\title{
Influence of the Hydrogen Adsorption to the Optical Properties of Boron Nitride Nanotubes
}

\author{
A.H. BAYANI* \\ Institute of Nanoscience and Nanotechnology, Kashan University, Kashan, Iran \\ (Received July 17, 2015; in final form February 19, 2016)
}

\begin{abstract}
In this research we have studied physisorption of hydrogen molecules on armchair $(3,3)$ boron-nitride nanotube using density functional methods. Optical properties of the boron-nitride nanotube, with and without adsorbed $\mathrm{H}_{2}$ molecules, were investigated under parallel and perpendicular polarized electric fields. The results indicate that the nanotube optical gap slightly changes due to $\mathrm{H}_{2}$ physisorption and increasing $\mathrm{H}_{2}$ physisorption suppresses the boron-nitride nanotube optical spectrum. Also, the nanotube gets more transparent as the $\mathrm{H}_{2}$ concentration increases, in other words boron-nitride nanotube dielectric function decreases. Anisotropic dielectric function is another result which is determined by random phase approximation method.
\end{abstract}

DOI: 10.12693/APhysPolA.129.348

PACS/topics: 78.67.Ch, 85.35.-p, 68.43.-h, 67.63.Cd, 67.80.ff

\section{Introduction}

After the first time that Dillon and co-workers reported adsorption of hydrogen molecule on carbon single-walled nanotubes [1], many experiments and theoretical studies have been done to understand the adsorption mechanism on nanotube structures. Recently, the hydrogen molecule reaction to nanostructures such as graphene and carbon nanotubes has been investigated more [2] and the results show these materials are suitable selections for hydrogen encapsulation. Also, more studies have shown that the binding energy of hydrogen molecules adsorption on the substrate can increase by hetero atoms insertion in nanostructures such as boron-nitride nanotubes (BNNT) or silicon-carbide nanotubes [3]. The studies shown that adsorption mechanism depends on hydrogen form and chemisorption occurs when hydrogen is adsorbed in atomic form [4]. On the other hand, if hydrogen is adsorbed in molecular form physisorption takes place and hydrogen molecule reacts with the nanostructure through Van der Waals force [5].

From the sensing point of view, nanostructures such as nanotubes, graphene, etc., have been illustrated to be promising nanoscale gas sensors due to their superb properties and large surface/volume ratio. Understanding changing of electronic and optical properties of these nanostructures due to gas adsorption outstandingly helps us to make gas sensors [6]. Generally, the electronic properties of sensors after exposing gas molecules will change, because of charge induction or charge transfer [7-9]. Molecule can act as a donor or acceptor of electron and causes a change in the Fermi energy of the system or it can add scattering channels [10]. Furthermore, the detailed knowledge of the optical properties of the

\footnotetext{
*e-mail: amirhossein.bayani@gmail.com
}

BNNTs under different gases are essential for their characterization and may be helpful to utilize them as nanooptoelectronic devices such as optoelectronic sensors or field effect transistor [11]. The BNNTs are expected to show several electronic, optical and magnetic properties such as the Coulomb blockade, photoluminescence and super paramagnetism [12]. In general, dielectric properties of nanomaterials are sensitive to size, dimensionality and morphology. Although the dielectric function of not exposed to gases BNNTs are calculated [13], there is no survey on optical spectra of the BNNTs under different gases such as hydrogen.

In this paper, we carry out first-principle calculations to obtain binding energy of hydrogen molecules physisorption on armchair $(3,3)$ boron-nitride nanotube. To examine changes in the dielectric function of the nanotube, optical spectra are computed under electric fields polarized both parallel and perpendicular to the nanotube axis.

\section{Calculation method}

The first-principle calculations were performed using density functional method in which the functional of exchange-correlation is Perdew-Burke-Ernzerhof (PBE) [14, 15]. Only behaviour of valence electrons was considered and interaction with ionic nucleus within norm-conserving pseudopotential [16] has been done. The calculations of optimized system, binding energy and charge transfer were carried out with the PWSCF code of the Quantum Espresso package [17], within plane waves (periodic boundary condition (PBC)) and pseudopotential. Orbitals of Kohn-Sham in plane-wave form up to 150 Ry have been developed. The SIESTA package [18] within LCAO approximation was used to obtain optical characteristic. For modelling the detection zone, an armchair BNNT $(3,3)$ which consisted of 18 boron and 18 nitrogen atoms have been used as a unit cell. Bond length between two atoms, before relaxation of the structure, was $1.45 \AA$ (Fig. 1). 


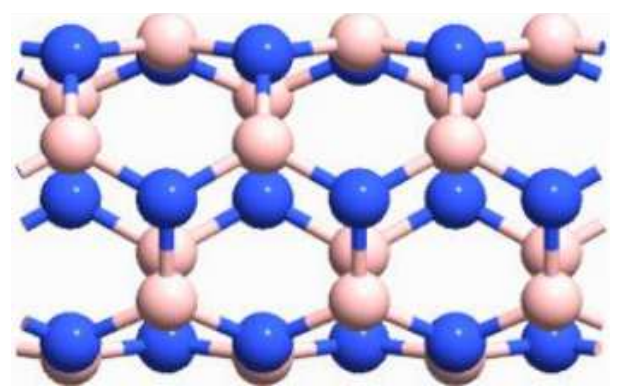

Fig. 1. Equilibrium geometries of boron-nitride nanotube $(3,3)$. Pink balls represent boron atoms and blue balls represent nitrogen atoms.

Symmetry cell was chosen a hexagonal lattice with parameters $a=b=25 \AA$ and $c=7.36 \AA$. We have selected $a$ and $b$ very large to avoid any interaction between periodic replica nanotubes. We have made the structure relaxed in the first Brillouin zone with $1 \times 1 \times 11$ sampling. For binding energy calculation and electronic structure, sampling in the first Brillouin zone $1 \times 1 \times 101$ has been done, where the Monkhorst-Pack method [19] has been used in all cases. The structure has been completely relaxed according to the Hellmann-Feynman theory [20] until maximum residual forces on all ions were less than $0.01 \mathrm{eV} / \AA$. The total energy also has been calculated with $10^{-7} \mathrm{eV}$ order of accuracy. Binding energy of hydrogen molecule $\left(E_{\mathrm{ad}}\right)$ was obtained through the difference between total energy of the nanotube plus hydrogen molecule(s) $\left(E_{\mathrm{BNNT}+\mathrm{nH} 2}\right)$ and sum of the total energy of isolated nanotube $\left(E_{\mathrm{BNNT}}\right)$ and isolated hydrogen molecule(s) $\left(n E_{\mathrm{H} 2}\right)$ under equal conditions (Eq. (1)):

$$
E_{\mathrm{ad}}=E_{\mathrm{BNNT}+\mathrm{nH} 2}-\left(E_{\mathrm{BNNT}}+n E_{\mathrm{H} 2}\right) / n,
$$

where $n$ denotes the number of molecules adsorbed on the BNNT. The concentration density of adsorbed $\mathrm{H}_{2}$ on BNNT is described by varying the number of $\mathrm{H}_{2}$ molecules in the detection zone, which is determined by the $\mathrm{H}_{2}: \mathrm{N}$ ratio based on the number of $\mathrm{H}_{2}$ molecules and the number of $\mathrm{N}$ (nitrogen) atoms on BNNT side wall.

The total charge transfer from the BNNT to the molecules can be estimated by projecting the charge density onto the atomic orbitals. The charge transfer was calculated as the difference between the Löwdin charges for pristine BNNT and BNNT with the adsorbate molecules. According to charge transfer, one can find out whether the adsorbate molecule acts as an acceptor or as a donor. It is important to mention that the charge transfer amount depends slightly on the method chosen for calculations.

To investigate the optical properties of the BNNT, the SIESTA package has been used which derive dipolar approximation to calculate the real and imaginary parts of the dielectric function, $\varepsilon(\omega)=\varepsilon_{1}(\omega)+\mathrm{i} \varepsilon_{2}(\omega)$ where $E=\hbar \omega$ being the energy of the incident radiation. The dielectric function is obtained by random phase approximation (RPA). We have set an energy range $[0,10] \mathrm{eV}$, Gaussian broadening of $0.005 \mathrm{eV}$ and a $1 \times 1 \times 201 k$-mesh.

\section{Results and discussion}

At first, the stable configuration for $\mathrm{H}_{2}$ adsorption on BNNT was determined. According to articles [5, 21, 22] and the existence of charge symmetry, the most stable state of the molecule is above the hexagonal centre of the nanotube. Therefore the hydrogen molecule was considered in two directions, perpendicular and parallel to the nanotube axis (Fig. 2).
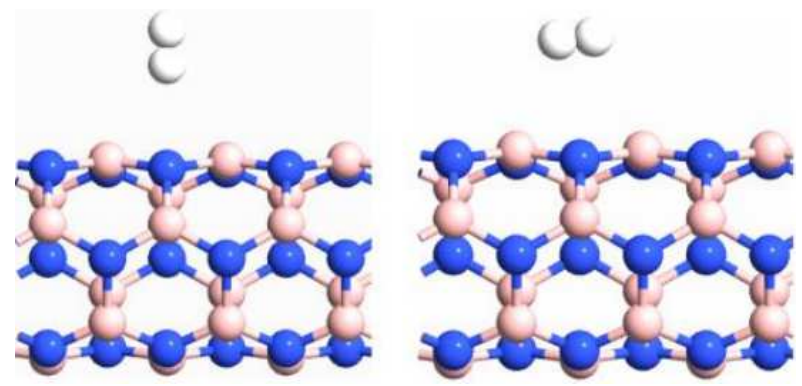

Fig. 2. Two different positions in studying hydrogen molecule on boron-nitride nanotube. White balls represent hydrogen atoms.

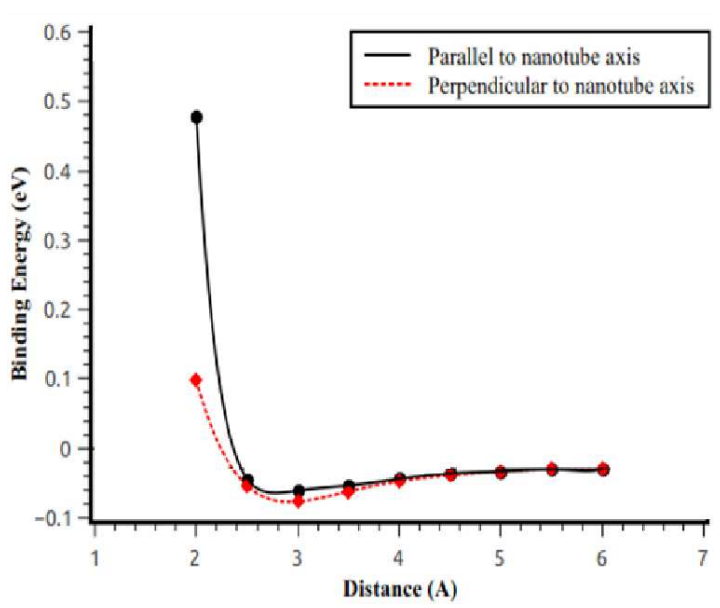

Fig. 3. Binding energy versus distance curve for two states (perpendicular and parallel to the nanotube axis).

Binding energy of the molecule, by applying Eq. (1), was obtained through changing the distance of hydrogen molecule from the surface of nanotube for 2 to $6 \AA$. The binding energy versus distance, for both perpendicular and parallel states, is shown in Fig. 3.

The binding energy of the perpendicular state is more than the parallel state so the perpendicular state is more stable. This shows that the polarization of hydrogen molecule in external field of the nanotube, depends on molecule direction and the external field of the BN nanotube. When the molecule is perpendicular to the nanotube axis, the external field of BNNT and the molecule are parallel, as a result the polarity of molecule and binding energy in this case are more than in the other case.

The minimum of the curve occurs at $2.9 \AA$ which is the equilibrium distance (ED) of the molecule from the 
surface of the nanotube. The binding energy (BE) corresponding to ED is approximately equal to $80 \mathrm{meV}$ and is less than the result reported in paper [3] as $\mathrm{BE}=110 \mathrm{meV}$ $(\mathrm{ED}=3.0 \AA)$.

The difference between the binding energy which is obtained here and obtained in paper [3] returns to nanotubes type. A zig-zag boron-nitride nanotube $(10,0)$ was considered in that paper whereas, an armchair boronnitride nanotube $(3,3)$ is studied here.

Then the concentration density is increased by adding $\mathrm{H}_{2}$ molecules uniformly to the symmetric sites on the tube side wall. Figure 4 shows the representative configurations of the BNNTs with different $\mathrm{H}_{2}: \mathrm{N}$ ratios.

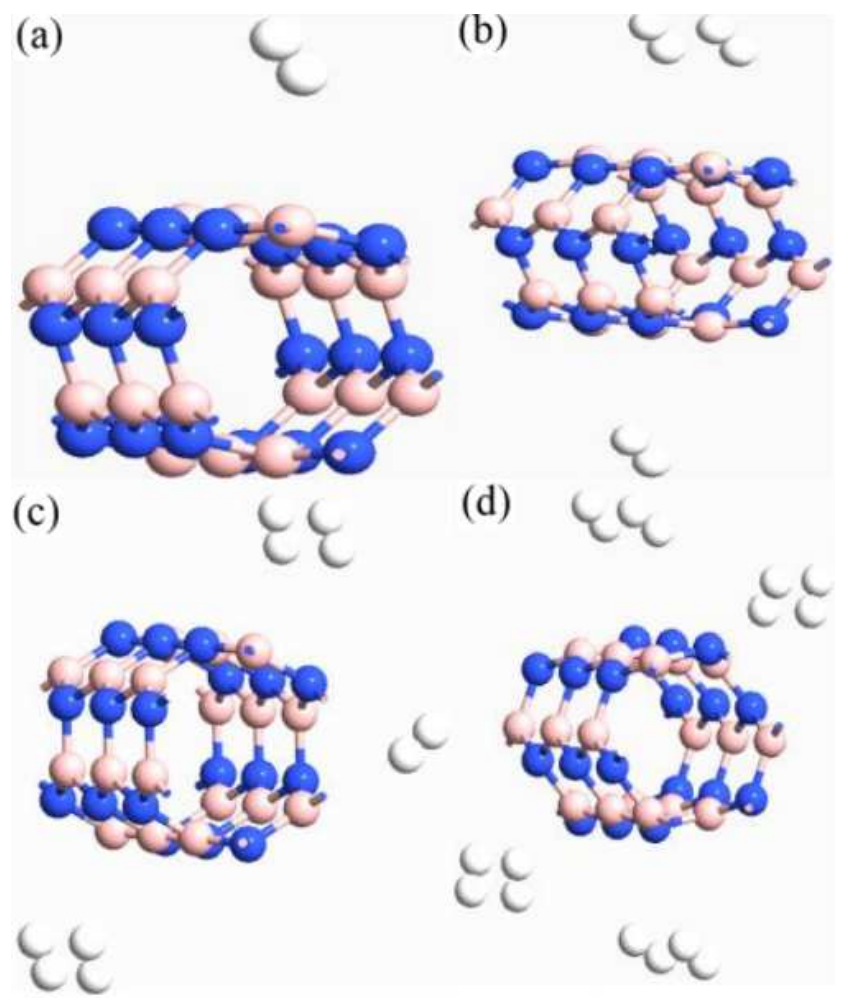

Fig. 4. Configurations of the BNNT with $\mathrm{H}_{2}: \mathrm{N}$ ratio of (a) $5.55 \%$, (b) $16.66 \%$, (c) $27.77 \%$, and (d) $44.44 \%$.

Binding energy and charge transfer were calculated for each concentration density and Table I presents the results. These results reveal that the increase in the $\mathrm{H}_{2}$ molecule concentration increases the binding energy and the charge transfer from the nanotube to the molecule(s).

TABLE I

Calculated adsorption binding energies (BE) and charge transfer $(\mathrm{CT})$.

\begin{tabular}{c|c|c}
\hline \hline $\mathrm{H}_{2}: \mathrm{N} \mathrm{Ratios}$ & BE $[\mathrm{meV}]$ & $\mathrm{CT}[e]$ \\
\hline $5.55 \%$ & 80.3 & 0.002 \\
$16.66 \%$ & 82.6 & 0.006 \\
$27.77 \%$ & 84.1 & 0.015 \\
$44.44 \%$ & 85.5 & 0.033
\end{tabular}

\subsection{Optical properties}

To investigate the optical properties of the BNNT, the real and imaginary parts of the nanotube dielectric function were computed for parallel ( $z$-direction) and perpendicular ( $x$-direction) polarization relative to the nanotube axis which are shown in Fig. 5.
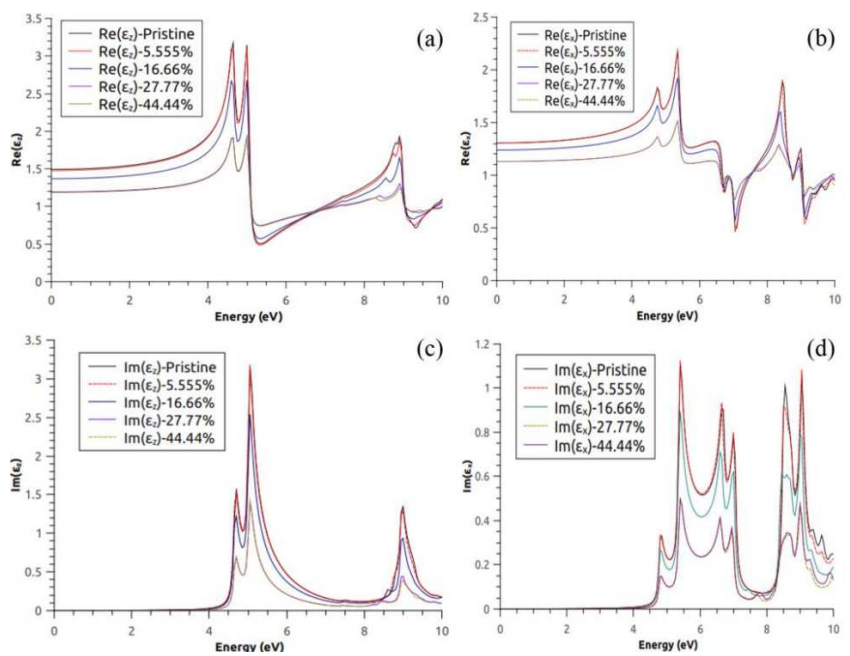

Fig. 5. The real $(\mathrm{a}, \mathrm{b})$ and imaginary $(\mathrm{c}, \mathrm{d})$ parts of the dielectric function of $(3,3)$ BNNT for parallel $(z)(\mathrm{a}, \mathrm{c})$ and perpendicular $(x)(b, d)$ polarizations to tube axis.

The imaginary part of the dielectric tensor can be computed by the knowledge of the electronic band structure of the solid. In the limit of linear optics, nonspin polarized and within the RPA frame work, we can use Eq. (2) [23].

$$
\begin{aligned}
& \operatorname{Im} \varepsilon_{\alpha \beta}(\omega)=\frac{h^{2} e^{2}}{\pi m^{2} \omega^{2}} \sum_{c, \theta} \int \mathrm{d} k\left\langle c_{k}\left|P^{a}\right| \vartheta_{k}\right\rangle\left\langle\vartheta_{k}\left|P^{\beta}\right| c_{k}\right\rangle \\
& \quad \times \delta\left(\varepsilon_{c k}-\varepsilon_{\vartheta k}-\omega\right) .
\end{aligned}
$$

The corresponding real part is obtained from the imaginary part of the dielectric tensor components by the Kramers-Kronig transformation as Eq. (3).

$$
\operatorname{Re} \varepsilon_{\alpha \beta}(\omega)=\delta_{\alpha \beta}+\frac{2}{\pi} \mathrm{P} \int_{0}^{\infty} \frac{\omega^{\prime} \operatorname{Im} \varepsilon_{\alpha \beta}\left(\omega^{\prime}\right)}{\omega^{\prime 2}-\omega^{2}} \mathrm{~d} \omega^{\prime} .
$$

Figure 5 shows the calculated results for real and imaginary parts of dielectric function, according to Eq. (2) and (3), for different $\mathrm{H}_{2}$ concentrations. The imaginary part of the dielectric function, as displayed in Fig. 5c,d indicate that, there is no optical adsorption in the low-energy region $(0-4 \mathrm{eV})$ for both polarizations but for further energies, there is optical adsorption and strong anisotropy in the optical spectra. Figure 5a,b is used to calculate the dielectric constants. The optical gaps and dielectric constants of $(3,3)$ BNNT for different $\mathrm{H}_{2}$ concentration are obtained from Fig. 5 and summarized in Table II.

One can see from the results that the optical band gap of the nanotube increases slightly with increasing $\mathrm{H}_{2}$ 
TABLE II

The calculated optical gaps $\left(E_{\mathrm{g}}\right)$ and dielectric constant $\varepsilon(0)$ of $(3,3)$ BNNT with difference $\mathrm{H}_{2}$ concentration for both polarizations (parallel $(z)$ and perpendicular $(x)$ to the tube axis).

\begin{tabular}{|c|c|c|c|c|}
\hline $\begin{array}{l}\mathrm{H}_{2}: \mathrm{N} \\
\text { ratios }\end{array}$ & $\begin{array}{l}\text { Optical } \\
\text { gap, } \\
\text { Parallel, } \\
E_{\mathrm{g}}(z) \\
{[\mathrm{eV}]}\end{array}$ & $\begin{array}{c}\text { Optical gap, } \\
\text { perpendicular, } \\
E_{\mathrm{g}}(x) \\
{[\mathrm{eV}]}\end{array}$ & $\begin{array}{c}\text { Dielectric } \\
\text { constant } \\
\text { parallel } \\
\varepsilon_{z}(0)\end{array}$ & $\begin{array}{c}\text { Dielectric } \\
\text { constant } \\
\text { perpendicular } \\
\varepsilon_{x}(0)\end{array}$ \\
\hline pristine & 3.40 & 4.15 & 1.49 & 1.32 \\
\hline $5.55 \%$ & 3.46 & 4.18 & 1.47 & 1.30 \\
\hline $16.66 \%$ & 3.51 & 4.23 & 1.37 & 1.24 \\
\hline $27.77 \%$ & 3.77 & 4.37 & 1.19 & 1.13 \\
\hline $44.44 \%$ & 3.93 & 4.42 & 1.18 & 1.11 \\
\hline
\end{tabular}

concentration but this is vice versa for dielectric constant behaviour and the nanotube becomes more transparent by hydrogenation. The optical gap value for the electric perpendicular case is larger than the parallel one which is in very good agreement with other DFT calculations [13].

\section{Conclusion}

Binding energy and charge transfer for different $\mathrm{H}_{2}$ concentration on armchair boron nitride nanotube $(3,3)$ are found. The results showed that binding energy and charge transfer increased by increasing $\mathrm{H}_{2}$ concentration density. To investigate the optical properties of the BNNT with different $\mathrm{H}_{2}$ concentration, dielectric function of the nanotube is calculated for both polarizations, parallel and perpendicular to the tube axis. The results show that the dielectric function is anisotropic and dielectric constant decreases with increasing $\mathrm{H}_{2}$ concentration and the nanotube becomes more transparent.

\section{References}

[1] A.C. Dillon, K.M. Jones, T.A. Bekkedahl, C.H. Kiang, D.S. Bethune, M.J. Heben, Nature 386, 377379 (1997).

[2] D.C. Elias, R.R. Nair, T.M.G. Mohiuddin, S.V. Morozov, P. Blake, M.P. Halsall, A.C. Ferrari, D.W. Boukhvalov, M.I. Katsnelson, A.K. Geim, K.S. Novoselov, Science 323, 610613 (2009).

[3] S.H. Jhi, Y.K. Kwon, Phys. Rev. B 69, 245407 (2004).

[4] A.H. Bayani, N. Shahtahmassebi, F.D. Vahedi, Physica E 53, 168 (2013).
[5] F. Costanzo, P.L. Silvestrelli, F. Ancilotto, J. Chem. Theory Comput. 8, 1288 (2012).

[6] Y. Wang, J.T.W. Yeow, J. Sensors 2009, 493904 (2009).

[7] M. Dell'Angela, G. Kladnik, A. Cossaro, A. Verdini, M. Kamenetska, I. Tamblyn, S.Y. Quek, J.B. Neaton, D. Cvetko, A. Morgante, L. Venkataraman, Nano Lett. 10, 2470 (2010).

[8] L. Venkataraman, J.E. Klare, I.W. Tam, C. Nuckolls, M.S. Hybertsen, M.L. Steigerwald, Nano Lett. 6, 458 (2006).

[9] D.J. Mowbray, G. Jones, K.S.J. Thygesen, Chem. Phys. 128, 111103 (2008).

[10] J. Zhao, A. Buldum, Jie Han, Jian Ping Lu, Nanotechnology 13, 195 (2002).

[11] M. Radosavljevic, J. Appenzeller, V. Derycke, R. Martel, P. Avouris, A. Loiseau, J.L. Cochon, D. Pigache, Appl. Phys. Lett. 82, 4131 (2003).

[12] T. Oku, N. Koi, K. Suganuma, J. Phys. Chem. Solids 69, 1228 (2008).

[13] D.V. Fakhrabad, T. Movlarooy, N. Shahtahmassebi, Phys. Status Solidi B 249, 1027 (2012).

[14] J.P. Perdew, K. Burke, Y. Wang, Phys. Rev. B 54, 16533 (1996).

[15] J.P. Perdew, K. Burke, M. Ernzerhof, Phys. Rev. Lett. 77, 3865 (1996).

[16] N. Troullier, J.L. Martins, Phys. Rev. B 43, 1993 (1991).

[17] P. Giannozzi, S. Baroni, N. Bonini, M. Calandra, R. Car, C. Cavazzoni, D. Ceresoli, G.L. Chiarotti, M. Cococcioni, I. Dabo, A. Dal Corso, S. de Gironcoli, S. Fabris, G. Fratesi, R. Gebauer, U. Gerstmann, C. Gougoussis, A. Kokalj, M. Lazzeri, L. Martin-Samos, N. Marzari, F. Mauri, R. Mazzarello, S. Paolini, A. Pasquarello, L. Paulatto, C. Sbraccia, S. Scandolo, G. Sclauzero, A.P. Seitsonen, A. Smogunov, P. Umari, R.M. Wentzcovitch, J. Phys. Condens. Matter 21, 39550219 (2009).

[18] J.M. Soler, E. Artacho, J.D. Gale, A. Garcia, J. Junquera, P. Ordejon, D.S. Portal, J. Phys. Condens. Matter 14, 2745 (2002).

[19] H.J. Monkhorst, J.D. Pack, Phys. Rev. B 13, 5188 (1976).

[20] J.G. Esteve, F. Falceto, C.G. Canal, Phys. Lett. A 374, 819 (2010).

[21] T.L. Pham, P.V. Dung, A. Sugiyama, N.D. Duc, T. Shimoda, A. Fujiwara, D.H. Chi, Comput. Mater. Sci. 49, S15 (2010).

[22] X. Wu, J. Yang, J.G. Hou, Q. Zhu, J. Chem Phys. 121, 8481 (2004)

[23] C. Ambrosch-Draxl, J.O. Sofo, Comput. Phys. Commun. 175, 1 (2006). 